\title{
Information Sharing of Large Class using Social Media Tool called SUGI-KOTOBA
}

\author{
Naoto Mukai \\ Department of Culture-Information Studies, \\ School of Culture-Information Studies, \\ Sugiyama Jogakuen University \\ 17-3, Hoshigaoka-motomachi, Chikusa-ku \\ Nagoya, Aichi, Japan \\ Corresponding Author: nmukai@sugiyama-u.ac.jp
}

\begin{abstract}
This paper reports the management of a large university classroom which introduces a social media tool called SUGI-KOTOBA. Generally, a large classroom has some problems in education for both teachers and students. For example, it is hard for teachers to hear a broad range of opinions from students quickly and effectively. Moreover, students often are educated through one-sided lectures, and they tend to have a lapse of concentration during class. As a solution to such problems, we introduced a social media tool called SUGI-KOTOBA that is jointly developed with SETJAPAN CORPORATION Inc. The SUGI-KOTOBA enables to form a temporal anonymous group of participants to share information by using a pre-shared keyword. In the group, teachers and students can communicate with each other via their smartphones. The feedback from students about the SUGI-KOTOBA indicated that a feeling of involvement in the class is increasing.
\end{abstract}

Keywords: SUGI-KOTOBA, Social Media Tool, Online Questionnaire, Large Class Management.

\section{Introduction}

In recent years, ICT education has been catching people's attention in Japan. MEXT(Ministry of Education, Culture, Sports, Science, and Technology) has held the meeting on ICT utilization for education and established "A Vision for Education Information" in 2010 (http://www.mext.go.jp/b_menu/houdou/23/04/1305484.ht $\mathrm{m})$. In the vision, the utilization of ICT in places of learning is one of the pillars of information education.

Based on the vision, various social networking services (SNS) were introduced as an educational tool to promote a communication among teachers and students as a trial. For ex-ample, Morimura ${ }^{(2)}$ reports that immediacy of Facebook is useful to contact other persons in class as soon as possible. Tajima et al. ${ }^{(4)}$ indicated that the quality of discussion can be improved by using LINE to share the theme or movies for an upcoming class. Sato et al. ${ }^{(3)}$ introduced an online groupware called Cyboze Live to a practical class for artisanship and reveals that the high frequency of the online groupware has a strong correlation with students' academic results. Hasegawa et al. ${ }^{(1)}$ experimented with a social reading system called Libra which is an e-book equipped with the social networking functions, and its questionnaire result showed that positive responses occupied 85 percent of the total responses.

However, in the most of past trials, targets are small-group classes or practical classes, in addition, a PC is often needed per a student for a group to access the SNS. On the other hand, large-group classes have particular problems in education for both teachers and students. For example, it is hard to exchange opinions among participants, and students only receive passive lessons focused on rote memory. In order to solve such problems, we introduced a social media tool called SUGI-KOTOBA. The SUGI-KOTOBA enables to form a temporal anonymous group of participants in the large classroom in a simple way. All that's required to form a group is to share a keyword. There are some variations of the keyword setting on a case-by-case basis, for example, a keyword may be a key phrase of a lesson, an answer of a quiz, and so on. The 
participants in the group can communicate on-line with each other via their smartphones. As a result of the introduction of SUGI-KOTOBA, it turned out that the students actively participate in class compared to before.

This paper consists of the following sections. Section 2 explains various features of SUGI-KOTOBA. Section 3 shows the introduction process of SUGI-KOTOBA into a large class in my university. Section 4 verifies the effect of SUGI-KOTOBA based on the feedback from students. Finally, section 5 concludes this paper.

\section{SUGI-KOTOBA}

SUGI-KOTOBA is one of the smartphone applicati ons for education and developed from a collaboration of Sugiyama Jogakuen University (http://www.sugiyamau.ac.jp/) and SETJAPAN CORPORATION Inc. (http://w ww.setjapan. co.jp/). Moreover, SUGI-KOTOBA is cust omized version of the AI-KOTOBA (https://www.ai-kot oba.me/) that is an application to exchange text, image, video, and so on. SUGI-KOTOBA have a function of a questionnaire, e.g., closed question, multiple-choice question, and free description, in addition to the origin al version.

A unique point of the SUGI-KOTOBA is how to form a group to share information. All that's required is to share a keyword among participants. Generally, a teacher decides an adequate keyword in advance and notify the keyword of students in the beginning of the class. At this time, other personal information of participants, i.e., real name, phone number, e-mail address, and so on, are not required to form a group. Thus, anyone can easily participate in the group compared with other similar SNS. We think that this is an important factor to enhance the willingness of participants even if participants are students of the same university.

Figure 1 shows a screenshot of posting messages. Left-side balloons are comments from students, and right-side balloons are comments from a teacher. There is an input field of a comment at the bottom of the screen. The comments which users entered are shared on a common timeline among teachers and students. Figure 2 shows a screenshot of a closed question. There is a result of the question in percentage terms. Anyone, anytime, anywhere, can design original questions in various formats, and participants can answer the question in a moment. As you can see, the interface of SUGI-KOTOBA is very simple, thus, it is not necessary to teach students about its operating instructions on ahead.

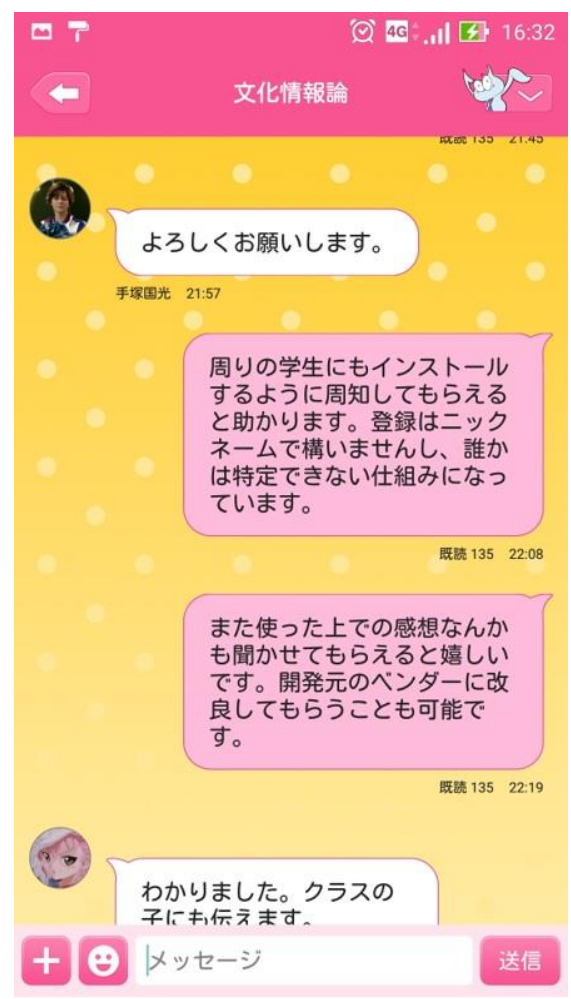

Fig. 1. Function of Posting Message

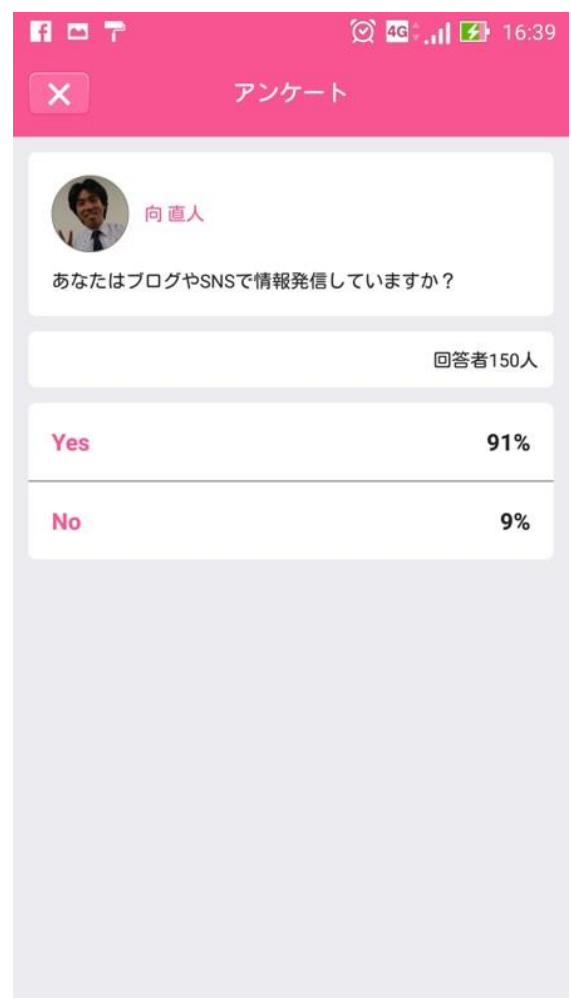

Fig. 2. Function of Questionnaire 


\section{Introduction Process to Large Class}

We introduced the SUGI-KOTOBA into the class of "INFORMATION AND CULTURE" in Sugiyama Jogakuen University on trial. The target of the class is first-year students, and the participants are 4 teachers (including the author of this paper) and 275 students. The main content of the class is a panel discussion with a topical theme by teachers. Before the introduction of the SUGI-KOTOBA, there were three intractable problems as following. We expected these problems to be solved by means of the introduction of SUGI-KOTOBA.

- It is difficult to hear opinions directly from students quickly and effectively.

- It tends to be one-sided lectures from teachers, and students often become poor concentration.

- The process of the discussion lacks flexibility.

First of all, we explained how to install the SUGI-KOTOBA to students via a portal site of the university about one month in advance. Students can download the install package of SUGI-KOTOBA for iOS and Android according to their smartphone models. As a result, 166 students (about 60\%) installed the SUGI-KOTOBA into their smartphones on the day of the panel discussion.

The process of the panel discussion is as following. Firstly, a teacher presents a common keyword to students to form a group of SUGI-KOTOBA. In this case, the common keyword is related to the theme of the panel discussion. Secondly, students watch a video related to the theme in Figure 3, and then teachers run a panel discussion regarding the video. During the panel discussion, a chair instructed students to answer four questions related to the theme by using SUGI-KOTOBA as shown in Figures 4. Students answer the questions via their smartphones in Figure 5. The results of the answers are promptly displayed on the screen in the front of the classroom in Figure 6. Finally, we receive the feedback about the SUGI-KOTOBA from students. The result of the feedback is described in the next section.

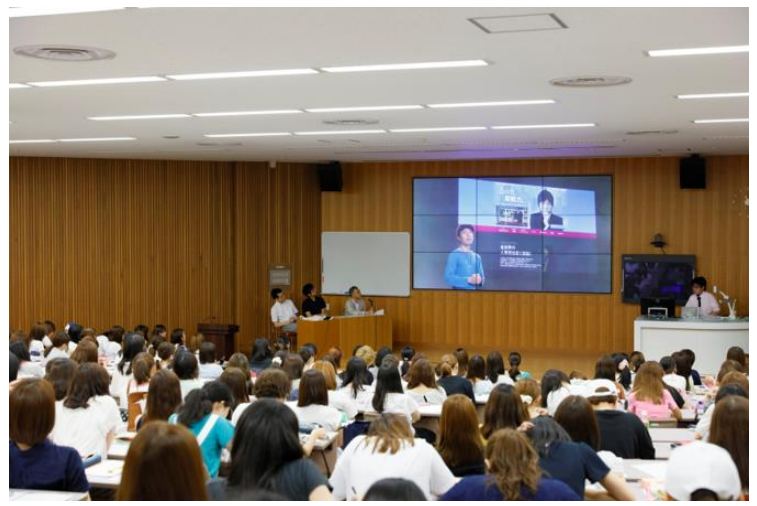

Fig. 3. Watching a video

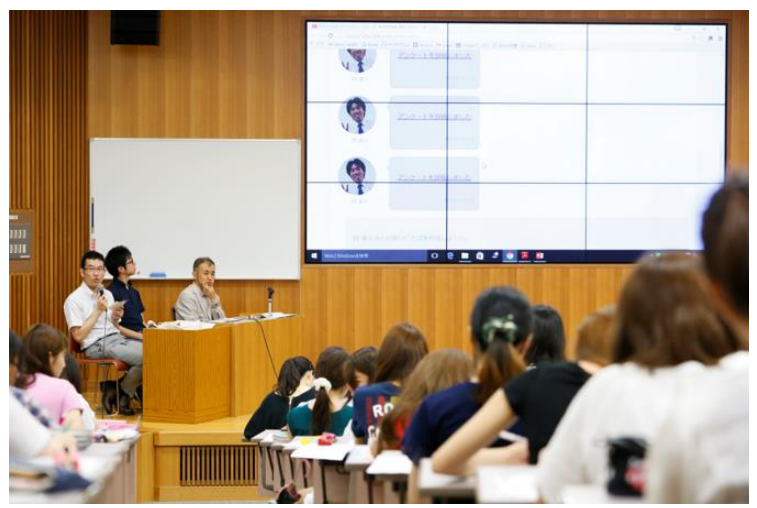

Fig. 4. Instruction of Questionnaire

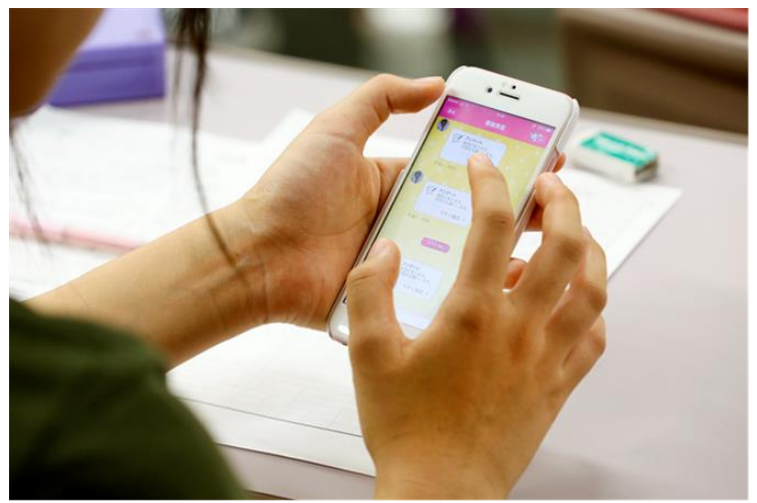

Fig. 5. Answering of questionnaire

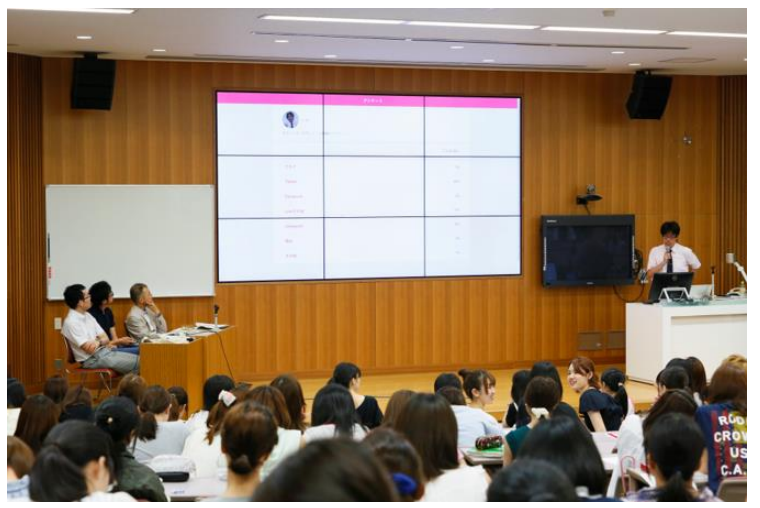

Fig. 6. Result of questionnaire 


\section{Feedback from Students}

After the panel discussion, we receive the feedback that consists of six questions about the SUGI-KOTOBA from students.

The first question is "Did you install SUGI-KOTOBA?", and its result is shown in Figure 7. Students who installed are only $73 \%$, and some of the others pointed the problem of installation method, e.g., capacity shortage of smartphones, communication failure of the classroom, and so on. We think these problems can be solved through the maintenance of the SUGI-KOTOBA.

The second question is "How about your sense of participation?", and its result is shown in Figure 8. Positive responses from students are $73 \%$. Some students answered that they find the SUGI-KOTOBA very interesting, but a few students felt uncomfortable using smartphones during class. Overall, it seems that most of the students participate the panel discussion actively from the viewpoint of teachers.

The third question is "How about your depth of understanding?", and its result is shown in Figure 9. Positive responses from students are 58\% which is less than the previous question. There is no strong dependence relationship between the depth of understanding and SUGI-KOTOBA.

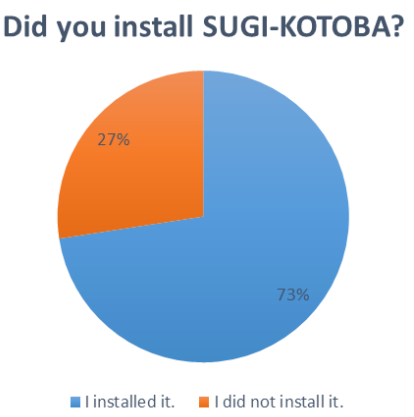

Fig. 7. Did you install SUGI-KOTOBA?

How about your sense of participation?

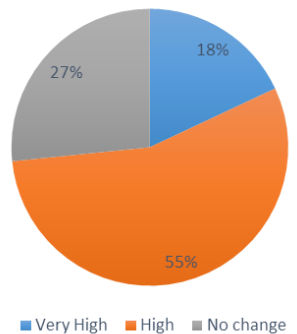

Fig. 8. How about your sense of participation?
The fourth question is "How about the feature of posting message?", and its result is shown in Figure 10. Positive responses from students are $48 \%$. The posting message is no new function, and it can be replaced by other social media tools. Thus, many students seem to think that this function is not special.

The fifth question is "How about the feature of questionnaire?", and its result is shown in Figure 11. Positive responses from students are 69\% which is more than the previous question. A questionnaire is an easy way to collect students' opinions for teachers, and the operation is fuss-free than the free description for students. Thus, many students responded favorably to the function of the questionnaire.

The last question is "Which is better, anonymous or real name?", and its result is shown in Figure 12. 66\% of students supported "anonymous". In the case of "anonymous", students can have a friendly discussion, but it does not need to have responsibilities for their words. There is no way that unfamiliar students are included in a Large-group class. Thus, we think "anonymous" is the better way than "real name" to encourage class's activity. At first, we thought that the SUGI-KOTOBA can be used as an attendance system by using "real name", but it isn't possible.

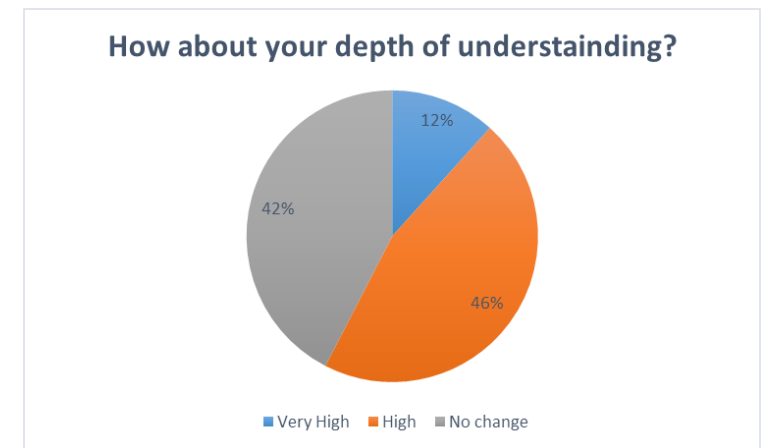

Fig. 9. How about your depth of understanding?

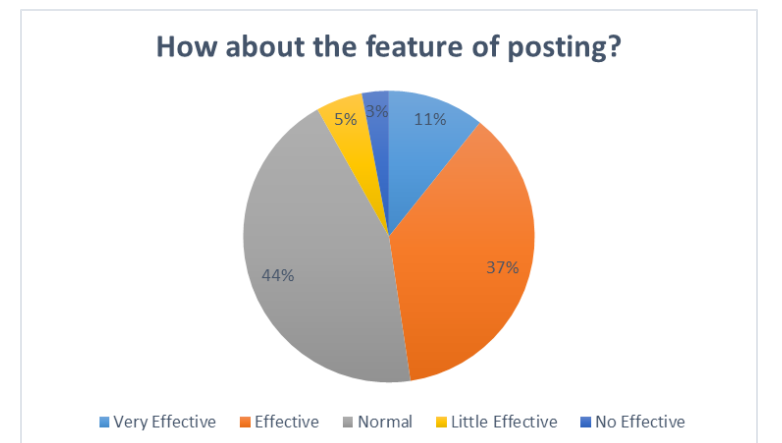

Fig. 10. How about the feature of posting a message? 


\section{Acknowledgment}

How about the feature of questionnaire?

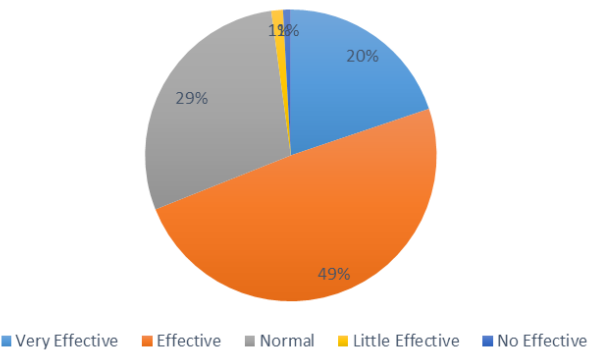

Fig. 11. How about the feature of the questionnaire?

Which is better, anonymous or real name?

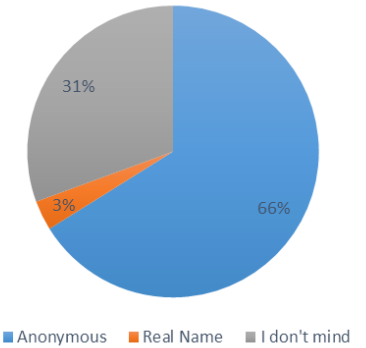

Fig. 12. Which is better, anonymous or real name?

Consequently, we can consider the introduction of SUGI-KOTOBA successful. Is is obvious that the sense of participation was high, and questionnaire via smartphone was effective than ever before.

\section{Conclusions}

In recent years, ICT education is closely watched in Japan. This move has inspired new education tools on the basis of SNS. In this paper, we reported the management of a large class which introduces the SUGI-KOTOBA which is a smartphone application for education. The feedback from students indicated that the SUGI-KOTOBA enables to promote an active class than before, and anonymous is the better way to realize a friendly discussion among students.

Our future work is to expand the introduction of SUGI-KOTOBA into other classes and other participants. However, in the case of other classes, there appeared to be a problem of cheating on the exam. Thus, we must consider available time and place of the SUGI-KOTOBA by case basis. Moreover, we would like to develop the SUGI-KOTOBA as a standard educational application in the world.
This paper was accomplished by courtesy of SETJAPAN CORPORATION Inc. We are grateful to Pres. Koji Nakao and Mr. Nozomu Kurachi of the company.

\section{References}

(1) S. HASEGAWA, A. YASUI, and M. YAMAGUCHI. Educational use of the social networking service and social learning. Journal of Nagoya Bunri University, 13:51-58, mar 2013.

(2) K. MORIMURA. Communication between teachers and students using social network service : Facebook-group fully utilized as a communication tool in and outside the classrooms. Proceedings of JSEE Annual Conference, 27(63):268-269, aug 2015.

(3) H. SATO, H. KIMURA, S. TAKANO, and R. AKAGI. A study of communicative development in engineering manufacturing practice : Practical examples of communicative development by using sns and learning portfolio. Proceedings of JSEE Annual Conference, 27(63):266-267, aug 2015.

(4) H. Tajima. A case study on education using a generalized sns for the management of a small class. Proceedings of FIT, 14(3):533-534, aug 2015. 